\title{
Anales de Biología
}

Volumen 34, 2012

\section{Evaluadores - Board of Advisor}

Queremos dar las gracias a todos los que se citan a continuación y otros revisores anónimos por dedicar su tiempo a evaluar los artículos de Anales de Biología vol. 34; su trabajo y dedicación es muy apreciada.

We would like to thank all those listed below and other anonymous reviewers for taking the time to review for Anales de Biología vol. 34; your work and dedication is much appreciated.

Dr. C. Aedo (Madrid)

Dr. T. Alcalde (Pamplona)

Dra. A. Andreu (Ensenada, Baja California, México)

Dr. I. Aranda (Madrid)

Dr. A. Bernabé (Murcia)

Dr. D. Boix (Gerona)

Dr. I. Bonilla (Madrid)

Dra. I. Buj (Zagreb, Croacia)

Dr. J.J. Camacho-Cristóbal (Sevilla)

Dra. R. Ma Camacho Ruiz (Guadalajara, Jalisco, México)

Dr. R. D. Campbell (Irvine, California, USA)

Dr. F. Casals (Lérida)

Dr. J. Casas (Almería)

Dr. J.J. Camacho-Cristóbal (Sevilla)

Dr. R.A. Carreno (Delaware, Ohio, USA)

Dr. J. Cuello (Murcia)

Dr. J.A. Del Río (Murcia)

Dr. L. Delgado (Salamanca)

Dr. J. A. Devesa Alcaraz (Cordoba)

Dr. M. Doucet (Cordoba, Argentina)

Dra. P. Drake Moyano (Cádiz)

Dra. A. Farrés (Mexico, D.F.)

Dr. D. Golani (Jerusalem, Israel)

Dra. A. B. Guarnaschelli (Buenos Aires, Argentina)

Dr. J. Juste (Sevilla)

Dr. E.T. Koutrakis (Kavala,Grecia)

Dr. R. Miranda (Pamplona)

Dr. J. Mota (Almería)

Dr. F. Oliva (Murcia)

Dr. A. Pérez Ruzafa (Murcia)

Dr. D. Rivera (Murcia)

Dra. M.J. Sánchez Ariza (Jaén)

Dr. P. Sánchez-Gómez (Murcia)

Dr. S. Talavera (Sevilla)

Dra. P. Torrente (Murcia)

Dr. J. Usera (Valencia) 